\title{
Clinicopathological features of epiretinal membranes in eyes filled with silicone oil
}

This article was published in the following Dove Press journal: Clinical Ophthalmology

\author{
Yoshiaki Tanaka' \\ Fumihiko Toyoda' \\ Machiko Shimmura-Tomita' \\ Nozomi Kinoshita \\ Hiroko Takano' \\ Yoh Dobashi ${ }^{2}$ \\ Shigeki Yamada ${ }^{2}$ \\ Hiroto Obata ${ }^{3}$ \\ Akihiro Kakehashi' \\ 'Department of Ophthalmology, \\ Saitama Medical Center, Jichi \\ Medical University, Saitama, \\ Japan; ${ }^{2}$ Department of Pathology, \\ Saitama Medical Center, Jichi \\ Medical University, Saitama, Japan; \\ ${ }^{3}$ Department of Ophthalmology, \\ Saitama Medical Center, Saitama \\ Medical University, Kawagoe, Japan
}

Correspondence: Akihiro Kakehashi Department of Ophthalmology, Saitama Medical Center, Jichi Medical University, I-847 Amanuma-cho, Omiya-ku, Saitama 330-8503, Japan

Tel +8I 486472111

Fax +8I 486485166

Email kakeaki@omiya.jichi.ac.jp
Purpose: The aim of this case series was to clarify the clinicopathological features of epiretinal membranes (ERMs) that developed in eyes after silicone oil (SO) tamponade to treat rhegmatogenous retinal detachments (RRDs).

Patients and methods: In the Department of Ophthalmology, Saitama Medical Center, Jichi Medical University, patients with idiopathic ERMs (23 eyes) and ERMs in eyes filled with SO (SO ERMs) after vitreous surgery to treat RRDs (nine eyes) were enrolled from July 2012 to March 2014. ERM tissues obtained intraoperatively were examined histopathologically. Besides the main outcome measure of the pathological findings of the ERM tissues, other outcome measures included the preoperative findings on optical coherence tomography (OCT) images and the surgical findings.

Results: Eight (89\%) of nine eyes with SO ERMs had bilayered membranes composed of a firm layer on the retinal side with glial cells and extracellular matrix and a fragile sponge-like layer on the vitreous side. The sponge-like layer was composed of emulsified SO surrounded by macrophages. Quantitative analysis showed that the areas with cluster of differentiation 68 (CD68)-positive macrophages identified by immunohistochemistry in eyes with SO ERMs were significantly $(P<0.001)$ larger than those in eyes with idiopathic ERMs. The findings on OCT images were consistent with the pathological features of the SO ERMs. Surgical removal of the SO ERMs was difficult because the sponge-like layer was fragile, and the underlying retina was also fragile due to inflammation. Conclusion: SO ERMs are bilayered membranes. Long-standing emulsified SO formed a sponge-like layer and SO (foreign body)-induced granulation and caused retinal inflammation in these eyes, making surgical removal difficult. A preoperative OCT examination is necessary to identify SO ERMs.

Keywords: epiretinal membrane, optical coherence tomography, pathological feature, rhegmatogenous retinal detachment, silicone oil, vitrectomy

\section{Introduction}

Some membranous proliferations on the macula, often referred to as epiretinal membranes (ERMs), can cause macular distortion and macular edema resulting in visual impairment and the need for surgical removal. In 1865, Iwanoff ${ }^{1}$ first described the abnormal proliferation of cellular membranes on the inner retinal surface.

ERMs develop under various conditions, eg, in normal eyes; in those with retinal detachments, chronic retinitis, or chorioretinitis; in eyes with central retinal vein occlusion and diabetic retinopathy; and after several procedures, such as scleral buckling, cataract extraction, retinal cryopexy, and laser retinal photocoagulation. Most ERMs are considered idiopathic and develop frequently in patients over 50 years of age. Several large clinical studies have reported that clinical prevalence rates range from $7 \%$ to $11.8 \%{ }^{2,3}$ ERMs can be composed of glia (astrocytes and Müller cells), retinal 
pigment epithelial cells, fibrous or myofibroblastic tissue, fibroinflammatory tissue, cortical vitreous, or any combination of these.

Since 1962, when Cibis et $\mathrm{al}^{4}$ introduced silicone oil (SO) for use in retinal surgery, controversy has existed over the role of SO and its potential toxicity. Emulsification is a wellknown phenomenon in SO-filled eyes. Other complications include cataract, band keratopathy, and secondary glaucoma that can potentially reduce visual acuity (VA). ${ }^{5-8}$

ERMs sometimes develop in eyes filled with $\mathrm{SO}$, such as after SO tamponade for proliferative vitreoretinopathy due to rhegmatogenous retinal detachments (RRDs). In the current study, we refer to them as ERMs in eyes filled with SO (SO ERMs). We have experienced difficulty in removing SO ERMs surgically compared with idiopathic ERMs. Minute hyperreflective areas located intraretinally, subretinally, and beneath the ERMs seen on spectral-domain optical coherence tomography (SD-OCT) images were found in eyes in which SO was used as a tamponade. ${ }^{9}$ The pathology of SO ERMs has been reported. ${ }^{10,11}$ However, to the best of our knowledge, no clinicopathological study of SO ERMs has been published. In the current study, we compared the clinicopathological features of SO ERMs with those of idiopathic ERMs.

\section{Patients and methods}

\section{Patients}

In the Department of Ophthalmology, Saitama Medical Center, Jichi Medical University, 64 patients (64 eyes) underwent a vitreous surgery to remove SO (5,000 centistokes) instilled during a primary vitreous surgery to treat RRDs. Of these 64 cases treated surgically from July 2012 to March 2014, SO ERMs were found in nine (14\%) patients (nine eyes) during an optical coherence tomography (OCT) examination performed 1 day before the surgery to remove the SO ERMs, and they were enrolled as the study group. Twenty-three patients (23 eyes) with idiopathic ERMs served as the control group, and the clinicopathological features of both groups were compared. Patients with other diseases, such as proliferative diabetic retinopathy and uveitis, were excluded from the study.

\section{Clinical examinations}

All patients underwent a detailed ophthalmologic examination preoperatively and postoperatively. The gender, age, preoperative and 3-month postoperative best-corrected VAs (BCVAs), and funduscopic and OCT findings were reviewed. The preoperative BCVA was measured, and the funduscopic and OCT examinations were performed 1 day before the surgery to remove the SO ERMs or idiopathic ERMs. The BCVA was measured as the decimal VA and converted to the logarithm of the minimum angle of resolution (logMAR) VA for analysis. We reviewed the OCT findings using Cirrus HD-OCT Model 4000 (Carl Zeiss Meditec AG, Jena, Germany) and measured the thickness of the ERMs on the fovea centralis and the central retinal thickness (CRT).

\section{Surgery}

One experienced surgeon (AK) performed all surgeries using the Accurus ${ }^{\circledR} 23 \mathrm{G}$ surgical system (Alcon Laboratories, Inc., Fort Worth, TX, USA). During the surgeries in the study group, we removed the SO and then the ERMs and the internal limiting membranes (ILMs). During the surgeries in the control group, we removed the ERMs and ILMs. Indocyanine green was used to stain the ILMs. We defined the time required to remove ERMs and ILMs as the duration from touching to removing membranes. The time was recorded on the surgical videos.

\section{Pathological examinations}

ERMs obtained during vitreous surgery were examined histopathologically and immunohistochemically. Immunohistochemical (IHC) staining for glial fibrillary acidic protein (GFAP) and cluster of differentiation 68 (CD68) was performed using the avidin-biotin complex technique. Formalinfixed, paraffin-embedded tissues were sliced into $4 \mu \mathrm{m}$-thick sections and mounted on silane-coated slides. After the sections were deparaffinized, endogenous peroxidase activity was blocked by incubation with $1 \%$ hydrogen peroxide in methanol for 30 minutes. Heat-induced antigen retrieval was performed in $0.01 \mathrm{~mol} / \mathrm{L}$ citrate buffer $(\mathrm{pH}, 6.0)$ for 30 minutes followed by blocking of nonspecific binding with $1 \%$ BSA. The slides were incubated with the primary antibody for CD68 (mouse monoclonal antibody, Clone KPI; Leica Biosystems Newcastle Ltd., Newcastle, UK) and GFAP (mouse monoclonal antibody, Clone GA5; Leica Biosystems Newcastle Ltd) at a dilution of 1:500 at $4^{\circ} \mathrm{C}$ overnight. After incubation at room temperature for 30 minutes with biotinylated anti-mouse immunoglobulin G (Dako Denmark A/S, Glostrup, Denmark) as the secondary antibody, the sections were incubated with avidin/biotin peroxidase (1:200; Vectastain SBC Kit; Vector Laboratories, Burlingame, CA, USA) for 60 minutes. Colorization was performed with diaminobenzidine tetrahydrochloride (SigmaAldrich Co., St Louis, MO, USA).

All slides were counterstained with H\&E and dehydrated and mounted with Permount Mounting Medium (Sigma-Aldrich Co.). To quantify the regions in which 
GFAP- or CD68-positive cells were identified, we used the BZ-X700 digital microscope system (Keyence, Osaka, Japan). One high-resolution image of an entire specimen was created using the image stitching system of BZ-H3XD (Keyence). Quantitative analyses of the GFAP- and CD68positive regions, which we referred to as the immunopositive regions, were performed using the Hybrid Cell Count Module/BZ-H3C software (Keyence). The entire specimen was marked with magenta, and the immunopositive regions were marked with yellow over it. The color coding of the immunopositive and immunonegative regions can be selected freely in this software. The ratio of the immunopositive areas to the entire specimen was calculated automatically in each specimen.

\section{Outcome measures}

The main outcome measure was the pathological findings of the ERMs. Other outcome measures included the preoperative OCT findings, the time required to remove the ERMs and ILMs, and the frequency of intraoperative use of a diamonddusted sweeper (DDS).

\section{Statistical analyses}

For statistical analyses, we used the Excel Tokei 2006 software (Social Survey Research Information Co., Ltd., Tokyo, Japan). Fisher's exact test was used to analyze the contingency table test. The Mann-Whitney $U$-test was used for the numerical parameters test of non-normal distribution. $P<0.05$ was considered statistically significant.

\section{Ethics approval and informed consent}

Bioethics Committee for Clinical Research, Saitama Medical Center, Jichi Medical University, approved this study (study number: S17-111), which adhered to the tenets of the Declaration of Helsinki. All study and control participants provided written informed consent for collection of specimens preoperatively. However, the Bioethics Committee for Clinical Research did not require written informed consent from the participants for reviewing their medical records, because all data were anonymized upon collection.

\section{Results}

\section{Study group}

The SO ERM study group included nine eyes (nine patients: six men and three women; Table 1). The patient age (mean \pm SD) at the time of removal of the SO ERMs was 61.3 \pm 9.9 years (range, 38-72 years). The mean preoperative logMAR BCVA was 0.85 (Snellen equivalent,

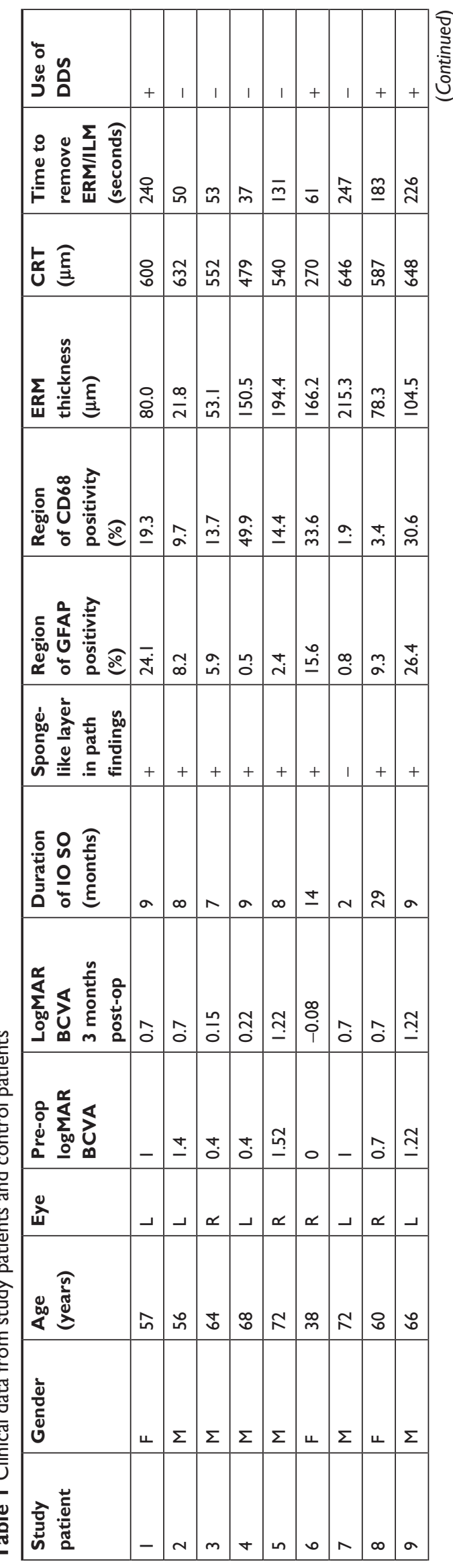




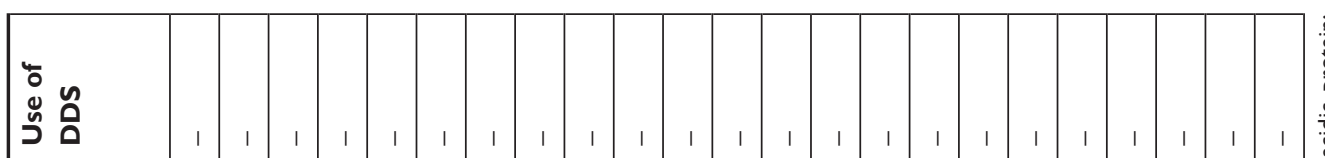

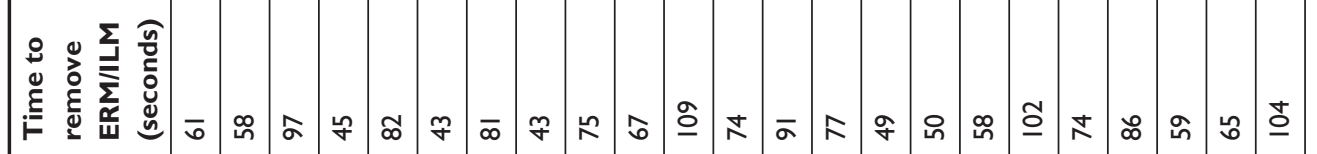

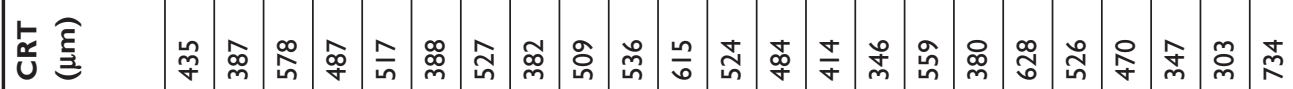

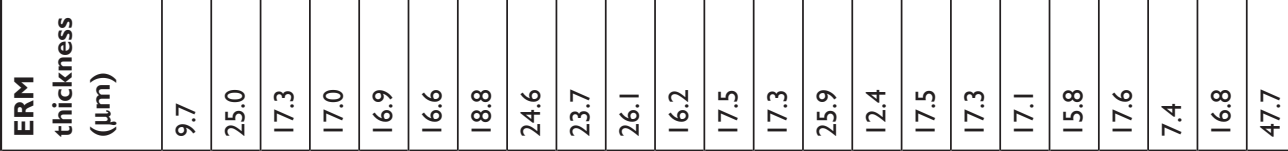

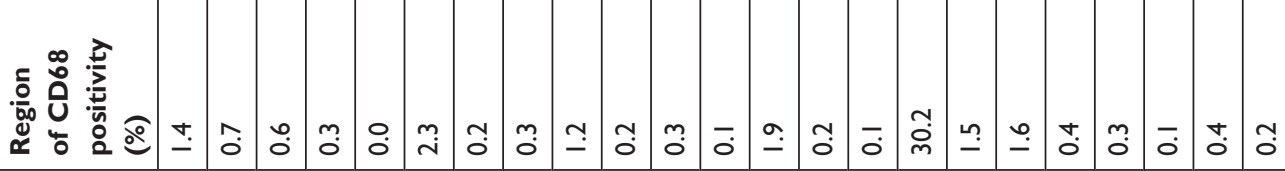

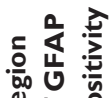

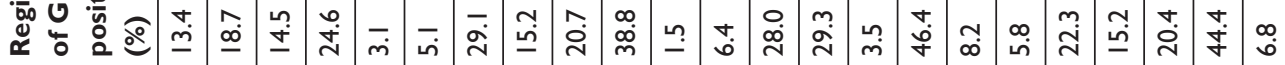

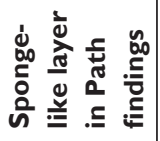

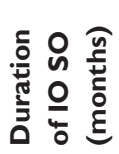

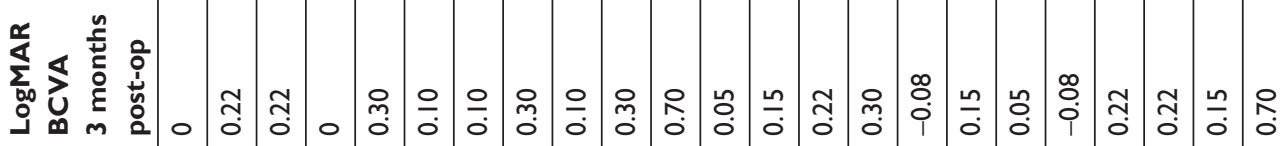

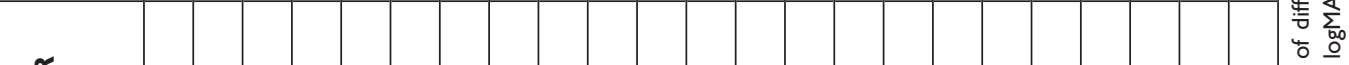

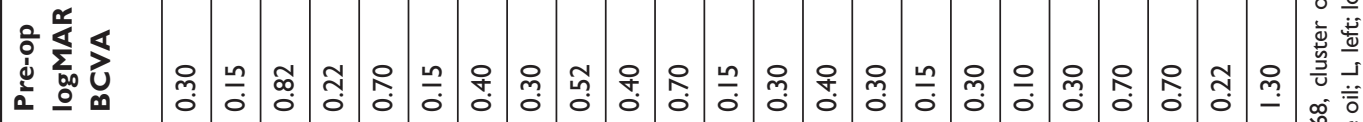

\begin{tabular}{|c|c|c|c|c|c|c|c|c|c|c|c|c|c|c|c|c|c|c|c|c|c|c|}
\hline 斊 & ــ & - & $\simeq$ & - & $\simeq$ & $\propto$ & - & 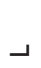 & - & - & ـ & - & ــ & $\simeq$ & $\propto$ & $\simeq$ & ـ & ـ & - & $\simeq$ & $\simeq$ & \lrcorner \\
\hline
\end{tabular}

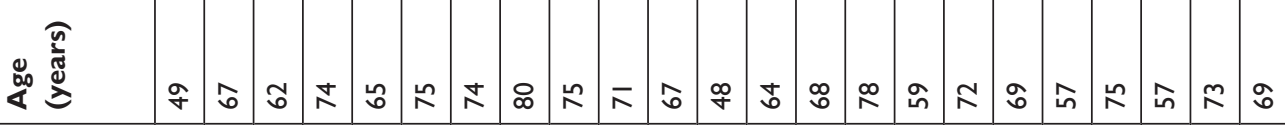
ปึ้

ชิ

$\frac{1}{\frac{1}{0}}$

$$
\text { ч } \Sigma \text { ᄂ }
$$


20/125-20/160) \pm 0.48 (range, 1.52-0). The mean postoperative $\log$ MAR BCVA at 3 months was 0.62 (Snellen equivalent, 20/80-20/100) \pm 0.42 (range, 1.22-0.08). In the study group, the mean period between SO tamponade and SO ERM

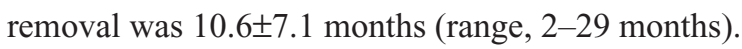

\section{Control group}

The idiopathic ERM control group included 23 eyes (23 patients: 13 men, 10 women; Table 1). The mean patient age in the control group was 67.3 \pm 8.5 years (range, $48-80$ years). The mean preoperative logMAR BCVA was 0.41 (Snellen equivalent, 20/50-20/63) \pm 0.28 (range, 1.30-0.10). The mean postoperative logMAR BCVA at 3 months was 0.19 (Snellen equivalent, 20/25-20/32) \pm 0.19 (range, 0.70-0.08).

\section{Pathological findings}

H\&E staining showed that the idiopathic ERMs had one layer of glial cells and an underlying extracellular matrix
(Figure 1A). The SO ERMs had two layers, ie, a glial cell/ extracellular matrix layer and a sponge-like layer (Figure 1B). The latter was composed of an emulsified SO bubble and was found in eight (89\%) of nine eyes with an SO ERM but not in the idiopathic ERM group $(P<0.001$, Fisher's exact test; Table 2).

GFAP is the standard marker of astrocytes. Glial cells immunostained for GFAP were visualized in the idiopathic ERMs and the SO ERMs (Figure 1C and 1D). Quantitative analysis indicated that the median area ratio of GFAP-positivity in the SO ERMs was $8.2 \%$ (range, $0.5 \%-26.4 \%$ ) and in the idiopathic ERMs 15.2\% (range, $1.5 \%-46.4 \% ; P=0.12$, Mann-Whitney $U$-test; Table 2 and Figure 2).

CD68 is particularly useful as a marker for the various cells of macrophage lineage. There are few CD68-positive cells in the idiopathic ERMs (Figure 1E). The spongelike layer in the SO ERMs was composed of emulsified $\mathrm{SO}$ in the form of granulomatous lesions surrounded by

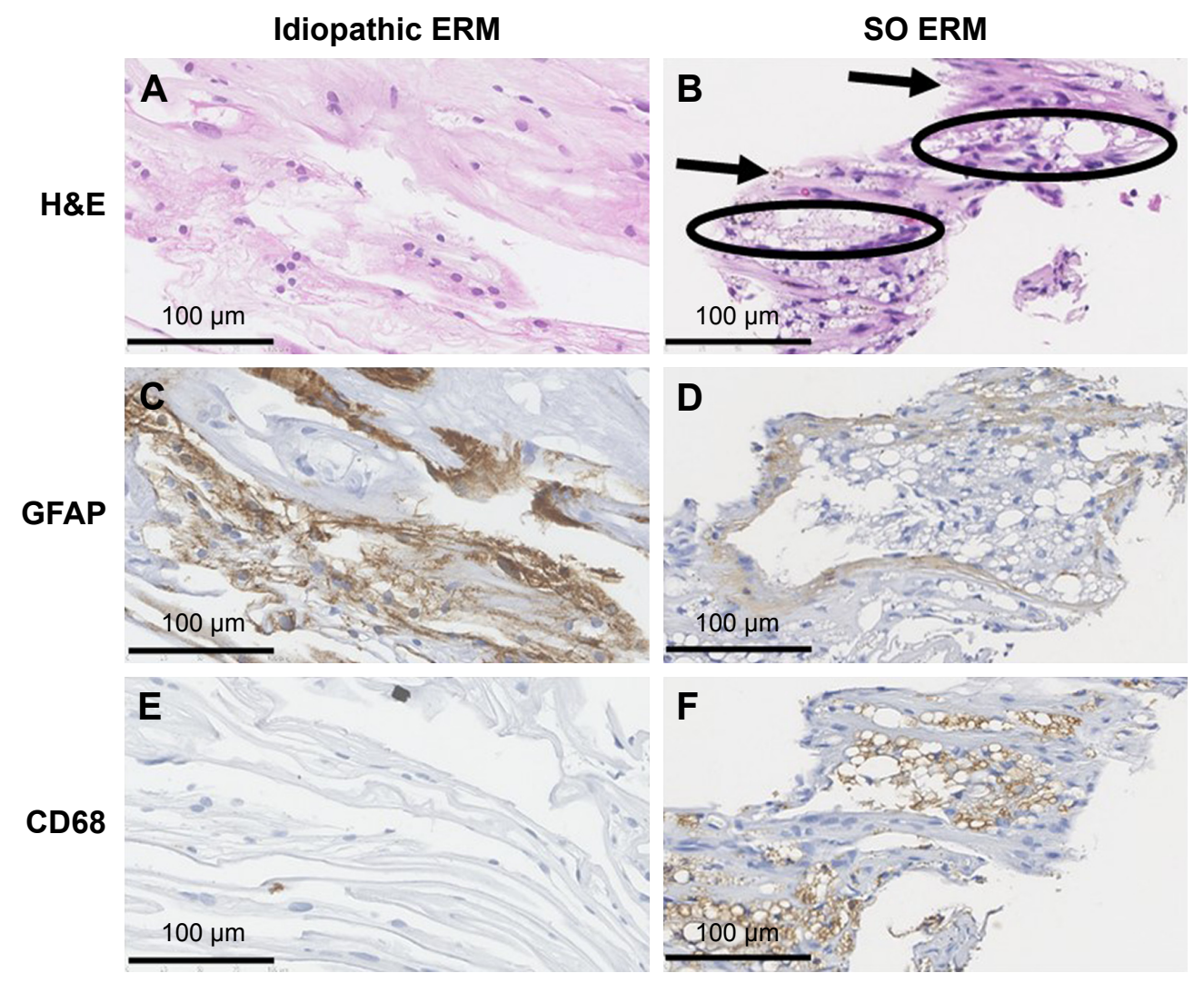

Figure I Microscopic images of H\&E staining and IHC staining of an idiopathic ERM and an SO ERM.

Notes: The pathological analysis shows that the idiopathic ERM has one layer of glial cells and extracellular matrix (A). The SO ERM has two layers (B). ie, the glial cell/ extracellular matrix layer (arrows) and the sponge-like layer (ellipses). The latter is composed of an emulsified SO bubble. Glial cells immunostained for GFAP are visualized in an idiopathic ERM and an SO ERM (C, D). There are few CD68-positive cells in an idiopathic ERM (E). The sponge-like layer in an SO ERM is composed of emulsified SO in the form of a granulomatous lesion surrounded by CD68-positive macrophages (F). Scale bars: $100 \mu \mathrm{m}$.

Abbreviations: CD68, cluster of differentiation 68; ERM, epiretinal membrane; GFAP, glial fibrillary acidic protein; IHC, immunohistochemical; SO, silicone oil; SO ERM, ERM in eyes filled with SO. 
Table 2 Clinical characteristics of the ERMs and SO ERMs in the study patients

\begin{tabular}{|l|l|l|l|}
\hline & Idiopathic ERM (23 eyes) & SO ERM (nine eyes) & $P$-value \\
\hline Sponge-like layer & $0 \%$ & $89 \%$ & $<0.00 \mathrm{I}^{\mathrm{a}, *}$ \\
\hline GFAP-positive region & $\mathrm{I} 5.2(1.5-46.4) \%$ & $8.2(0.5-26.4) \%$ & $0.12^{\mathrm{b}}$ \\
\hline CD68-positive region & $0.3(0-30.2) \%$ & $14.4(1.9-49.9) \%$ & $<0.00 \mathrm{I}^{\mathrm{b}, *}$ \\
\hline Thickness of ERM & $17.3(7.4-47.7) \mu \mathrm{m}$ & $104.5(21.8-215.3) \mu \mathrm{m}$ & $<0.00 \mathrm{I}^{\mathrm{b}, *}$ \\
\hline CRT & $487(303-734) \mu \mathrm{m}$ & $587(270-648) \mu \mathrm{m}$ & $0.042^{\mathrm{b}, * *}$ \\
\hline Time required to remove ERMs and ILMs & $74(43-109)$ seconds & $13 \mathrm{I}(37-247)$ seconds & $0.22^{\mathrm{b}}$ \\
\hline Frequency of use of DDS & $0 \%$ & $44 \%$ & $0.004^{\mathrm{a}, *}$ \\
\hline
\end{tabular}

Notes: Data are presented as median (range) unless otherwise indicated. aFisher's exact test was used. ${ }^{b}$ Mann-Whitney U-test was used. ${ }^{*} P<0.01 ; * * P<0.05$.

Abbreviations: CD68, cluster of differentiation 68; CRT, central retinal thickness; DDS, diamond-dusted sweeper; ERM, epiretinal membrane; GFAP, glial fibrillary acidic protein; ILM, internal limiting membrane; SO ERM, ERM in eyes filled with silicone oil.

CD68-positive macrophages (Figure 1F). Quantitative analysis indicated that the median area ratio of CD68 positivity in the specimen was $14.4 \%$ (range, $1.9 \%-49.9 \%$ ) in the SO ERMs and $0.3 \%$ (range, $0 \%-30.2 \%$ ) in the idiopathic ERMs $(P<0.001$, Mann-Whitney $U$-test; Table 2 and Figure 3).

\section{OCT findings}

OCT showed that the fovea centralis was thicker in the SO ERMs than in the idiopathic ERMs (median thickness in SO ERMs, $104.5 \mu \mathrm{m}$; range, $21.8-215.3 \mu \mathrm{m}$ vs idiopathic ERMs, $17.3 \mu \mathrm{m}$; range, 7.4-47.7 $\mu \mathrm{m} ; P<0.001$, Mann-Whitney $U$-test; Table 2 and Figure 4). The SO ERMs had two layers;

\section{Idiopathic ERM}

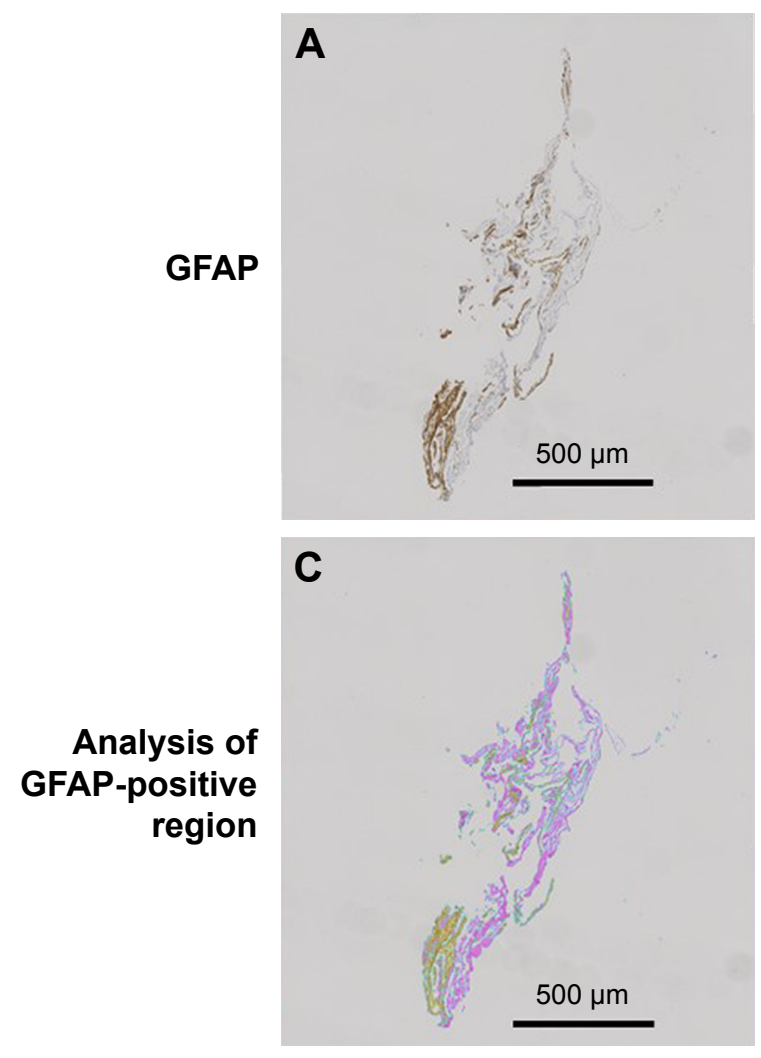

SO ERM
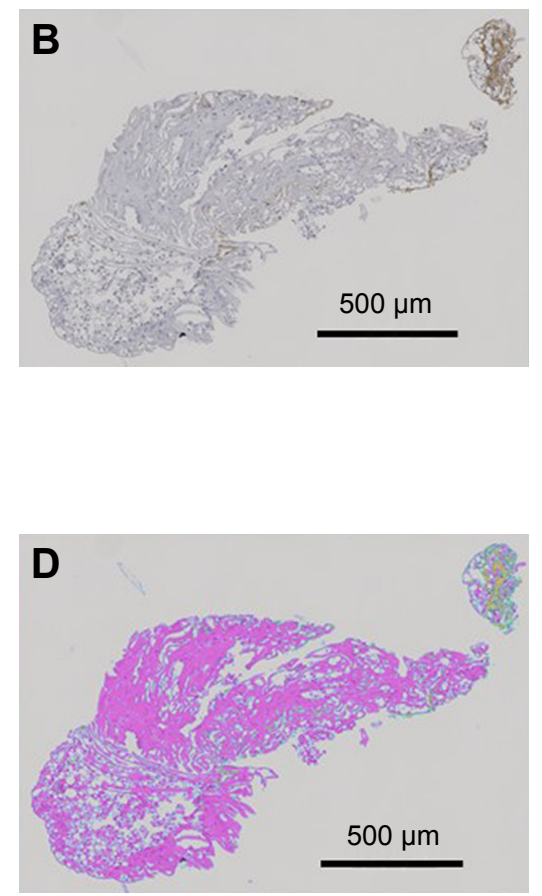

Figure 2 Analysis of GFAP-positive region.

Notes: Glial cells immunostained for GFAP are visualized in an idiopathic ERM and an SO ERM $(\mathbf{A}, \mathbf{B})$. The entire specimen is marked with magenta, and the immunopositive regions are marked with yellow over it (C, D). The color coding of the immunopositive and immunonegative regions can be selected freely with this software. The ratio of the immunopositive areas to the entire specimen is calculated automatically in each specimen. Quantitative analysis shows no significant differences between the region ratio of the GFAP positivity in an idiopathic ERM and an SO ERM. Scale bars: $500 \mu \mathrm{m}$.

Abbreviations: ERM, epiretinal membrane; GFAP, glial fibrillary acidic protein; SO ERM, ERM in eyes filled with silicone oil. 


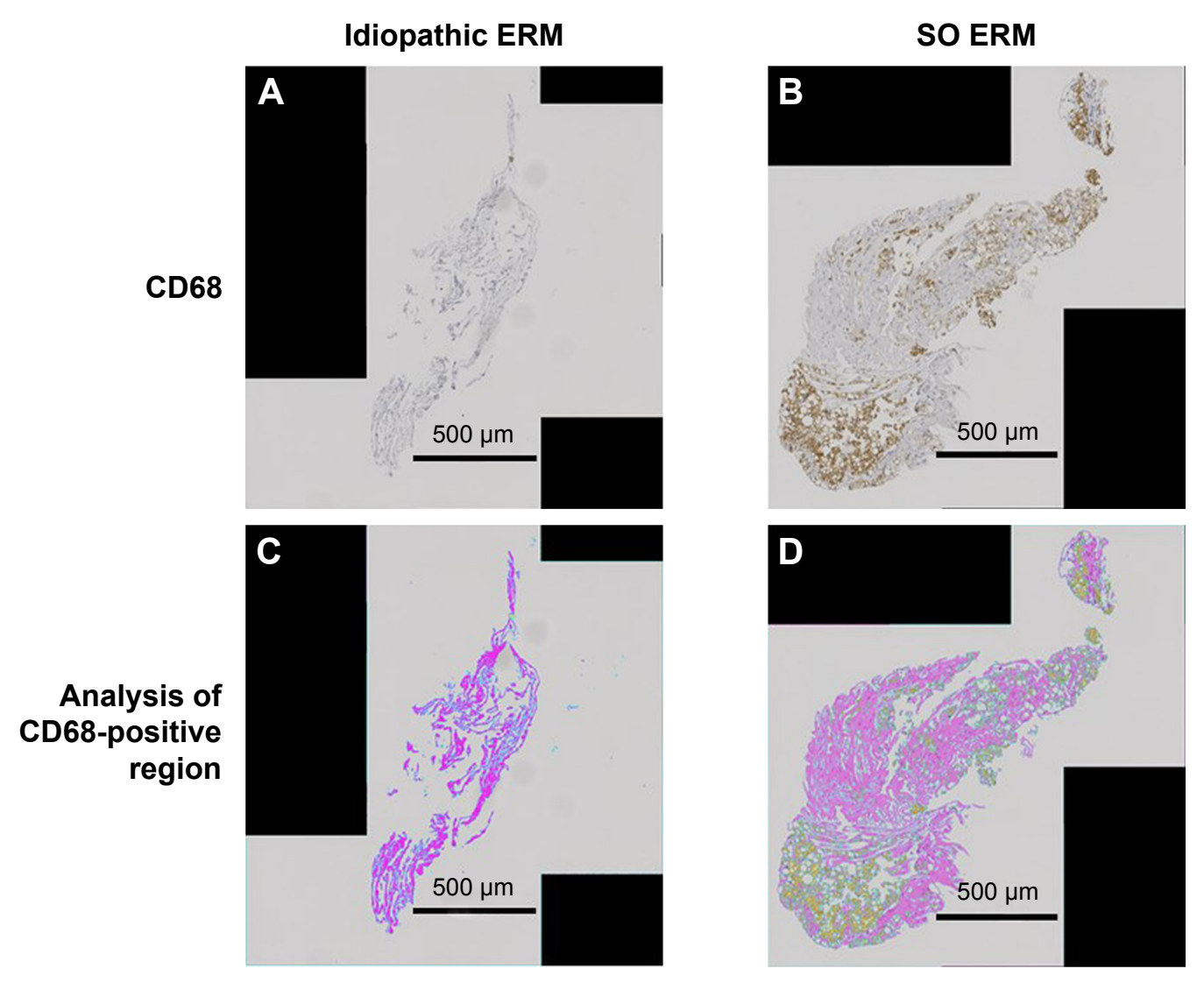

Figure 3 Analysis of $C D 68$-positive region.

Notes: There are few CD68-positive cells in an idiopathic ERM (A). The sponge-like layer in an SO ERM is composed of emulsified SO in the form of a granulomatous lesion surrounded by CD68-positive macrophages (B). The entire specimen is marked with magenta, and the immunopositive regions are marked with yellow over it (C, D). The color coding of the immunopositive and immunonegative regions can be selected freely with this software. The ratio of the immunopositive areas to the entire specimen is calculated automatically in each specimen. The region ratio of the CD68-positive macrophages in an SO ERM is significantly larger than in an idiopathic ERM. Scale bars: $500 \mu \mathrm{m}$. Abbreviations: CD68, cluster of differentiation 68; ERM, epiretinal membrane; SO ERM, ERM in eyes filled with silicone oil.

Idiopathic ERM
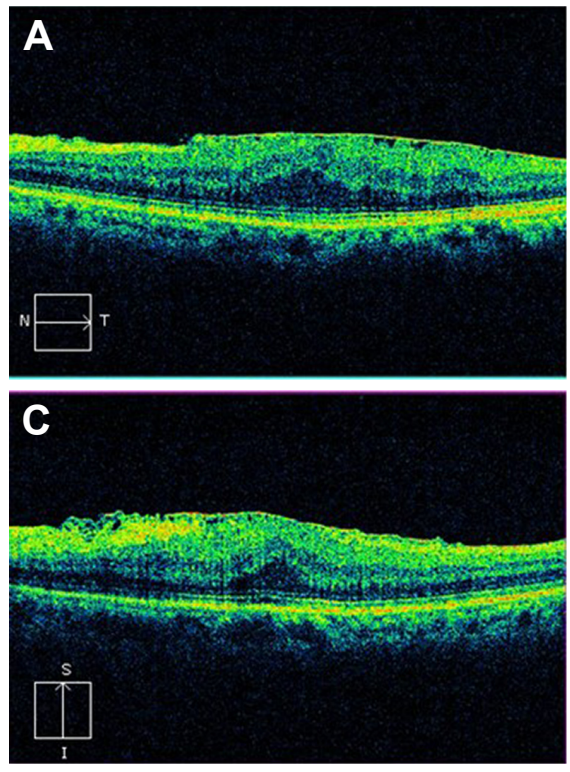

\section{SO ERM}
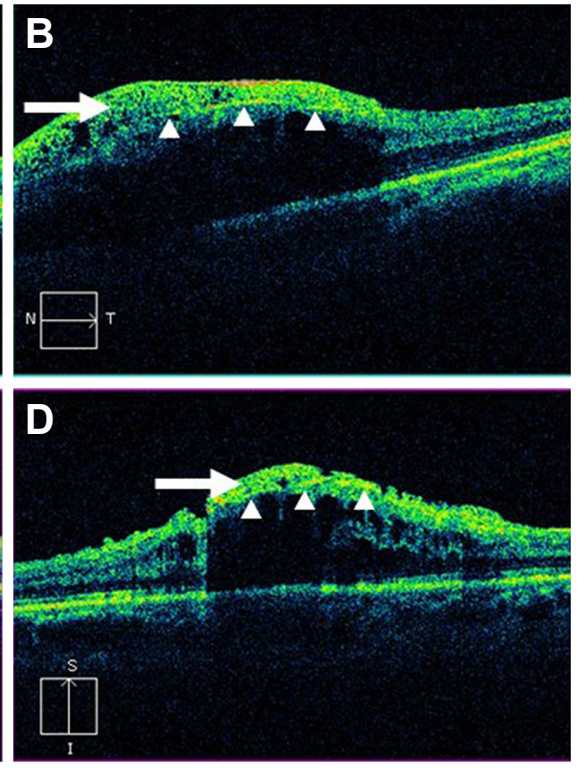

Figure 4 OCT findings in an idiopathic ERM and an SO ERM.

Notes: Compared with typical OCT images in the idiopathic ERM (A, C), the SO ERM is thicker and has two layers (B, D). The layer on the vitreous side of the SO ERM is composed of emulsified SO (arrows). The layer on the retinal side of the SO ERM is composed of glial cells and extracellular matrix (arrowheads). Severe retinal edema possibly resulting from inflammation is seen in the SO ERM (B, D).

Abbreviations: S, superior; I, inferior; N, nasal; T, temporal; ERM, epiretinal membrane; OCT, optical coherence tomography; SO, silicone oil; SO ERM, ERM in eyes filled with SO. 
the layer on the vitreous side of the SO ERMs had a granular appearance that corresponded to the granulomatous lesions containing emulsified SO, and the layer on the retinal side of the SO ERMs had a patchy appearance that corresponded to the glial cells and extracellular matrix. The median CRT of the SO ERMs was $587 \mu \mathrm{m}$ (range, 270-648 $\mu \mathrm{m}$ ) and that of the idiopathic ERMs was $487 \mu \mathrm{m}$ (range, 303-734 $\mu \mathrm{m}$; $P=0.042$, Mann-Whitney $U$-test; Table 2 and Figure 4). Severe retinal edema was found in the SO ERMs that possibly resulted from inflammation.

\section{Surgical findings}

The surgical findings indicated that the SO ERMs had two layers, but the idiopathic ERMs had one firm layer. The vitreous side of the SO ERMs was a sponge-like fragile layer; the retinal side was firm. The median time required to remove the ERMs and ILMs from eyes with SO ERMs was 131 seconds (range, 37-247 seconds), and the time to remove from eyes with idiopathic ERMs was 74 seconds (range, 43-109 seconds); the difference did not reach significance $(P=0.22$, Mann-Whitney $U$-test; Table 2$)$. A DDS was used to remove the layer on the vitreous side of the SO ERM before the layer on the retinal side of the SO ERM was removed in four (44\%) of nine eyes, but a DDS was not used to remove the idiopathic ERMs in any eyes $(P=0.004$, Fisher's exact test; Table 2). The retinas in eyes with an SO ERM were fragile because of severe retinal edema.

\section{Discussion}

Few studies have reported the OCT findings in eyes with SO tamponade. Chung and Spaide ${ }^{12}$ identified intraretinal SO vacuoles using the first-generation OCT and described intraretinal cystoid spaces in one patient who underwent macular hole surgery with ILM peeling and temporary SO tamponade. Errera et $\mathrm{al}^{9}$ reported minute hyperreflective areas located intraretinally, subretinally, and beneath the ERMs on SD-OCT images in eyes with SO tamponade. The authors concluded that the hyperreflective areas were likely to be very small bubbles of emulsified SO.

In the current study, OCT showed the structures that corresponded to the pathological features in eyes with SO ERMs. A bilayered membrane, glial cell/extracellular matrix layer, and sponge-like layer were found in the SO ERMs; the latter was granular and composed of emulsified SO. OCT showed that the sponge-like layer was on the vitreous side of the SO ERMs and the glial cell/extracellular matrix layer was on the retinal side of the SO ERMs. The current study is the first to describe the presence of a two-layered membrane in the SO ERMs. Since OCT has evolved and can detect minute changes in the vitreoretinal interface, a preoperative OCT examination is imperative for predicting pathological changes in the SO ERMs.

The specimens were so minute that we occasionally lost the orientation when they were embedded in paraffin. In addition, each specimen was partially curled. Therefore, histopathologically, the two layers of the membrane were intermingled but distinguishable based on H\&E staining and IHC staining.

Since elastic staining and other immunostaining were not performed in the current study, we could not determine whether the extracellular matrix was genuine collagen. However, Smiddy et $\mathrm{al}^{13}$ reported that the idiopathic ERMs contained varying proportions of four cell types, ie, retinal pigment epithelium (RPE), fibrous astrocytes, fibrocytes, and myofibroblasts. The extracellular matrix of idiopathic ERMs is composed primarily of collagen, ${ }^{14}$ which may have been produced from the RPE, fibroblasts, and myofibroblasts. The preretinal membranes following retinal detachments are inherently different from idiopathic ERMs. Michels ${ }^{15}$ estimated that roughly $10 \%$ contain vessels, but nearly all idiopathic ERMs are avascular. More importantly, the preretinal membranes after RRDs tend to merge along a spectrum with proliferative vitreoretinopathy. A much better control group would have included ERMs following RRD surgery which were not exposed to SO. However, cases of ERM after RRD surgery without SO tamponade are very rare in our clinic possibly because the premacular vitreous cortex usually is removed meticulously in those cases without SO tamponade.

CD68 immunostaining showed that the macrophages that surrounded the emulsified SO occurred more frequently in the SO ERMs than in the idiopathic ERMs. Quantitative analysis identified significant differences in the immunopositive regions between the two study groups, indicating phagocytosis of the emulsified SO by the macrophages. Heidenkummer et $\mathrm{al}^{10}$ and Wickham et $\mathrm{al}^{11}$ reported the presence of macrophages in SO ERMs. The current results supported the findings of those studies. In the current SO ERM group, the mean interval between the last vitreous surgery and SO removal was 10.6 \pm 7.1 months (range, 2-29 months). The long-standing emulsified SO was composed of a sponge-like layer that might cause retinal inflammation in eyes with $\mathrm{SO}$ ERMs. We did not have a long duration of observation and periodic OCT images in each case. Therefore, we could not evaluate the relationship between the development of SO ERMs and the changes in the foveal thickness. However, it is obvious that the SO ERMs should be removed before the bilayered membranes develop. 
Removing the SO ERMs required more time than removing the idiopathic ERMs, but the time difference did not reach significance. Surgical removal of the SO ERMs was difficult because the sponge-like layer was fragile, and the underlying retina was also fragile due to inflammation. The vitreous side of the SO ERMs was a fragile sponge-like layer that crumbled easily when grasped; in contrast, the retinal side was firm. Therefore, we used a DDS to sweep the fragile sponge-like layer. After removing that layer, we grasped the firm layer on the retinal side and removed it.

\section{Conclusion}

SO ERMs are bilayered membranes. Long-standing emulsified SO formed a sponge-like layer and SO (foreign body)induced granulation and caused retinal inflammation in these eyes, making surgical removal difficult. A preoperative OCT examination is necessary to identify SO ERMs. We emphasize that the bilayered membrane should be removed carefully, because the retina is edematous and fragile as a result of severe inflammation.

\section{Abbreviations}

BCVA, best-corrected visual acuity; CD68, cluster of differentiation 68; CRT, central retinal thickness; DDS, diamonddusted sweeper; ERM, epiretinal membrane; GFAP, glial fibrillary acidic protein; IHC, immunohistochemical; ILM, internal limiting membrane; OCT, optical coherence tomography; RRD, rhegmatogenous retinal detachment; SD-OCT, spectral-domain optical coherence tomography; SO, silicone oil; SO ERM, ERM in an eye filled with SO; VA, visual acuity; logMAR, logarithmic minimum angle of resolution.

\section{Acknowledgments}

We presented our results at the American Academy of Ophthalmology annual meeting in 2015. The authors would like to thank Yoko Noguchi for their research assistance. There was no financial support for this study.

\section{Disclosure}

The authors report no conflicts of interest in this work.

\section{References}

1. Iwanoff A. Beiträge zur normalen und pathologischen Anatomie des Auges [Contributions to the normal and pathological anatomy of the eye]. Albrecht von Graefes Archiv für Ophthalmologie. 1865;11(1):135-170.

2. Mitchell P, Smith W, Chey T, Wang JJ, Chang A. Prevalence and associations of epiretinal membranes. The Blue Mountains Eye Study, Australia. Ophthalmology. 1997;104(6):1033-1040.

3. Klein R, Klein BE, Wang Q, Moss SE. The epidemiology of epiretinal membranes. Trans Am Ophthalmol Soc. 1994;92:403-425; discussion 425-430.

4. Cibis PA, Becker B, Okun E, Canaan S. The use of liquid silicone in retinal detachment surgery. Arch Ophthalmol. 1962;68:590-599.

5. Watzke RC. Silicone retinopiesis for retinal detachment. A long-term clinical evaluation. Arch Ophthalmol. 1967;77(2):185-196.

6. Foulks GN, Hatchell DL, Proia AD, Klintworth GK. Histopathology of silicone oil keratopathy in humans. Cornea. 1991;10(1):29-37.

7. Abrams GW, Azen SP, McCuen BW 2nd, Flynn HW Jr, Lai MY, Ryan SJ. Vitrectomy with silicone oil or long-acting gas in eyes with severe proliferative vitreoretinopathy: results of additional and long-term follow-up. Silicone Study report 11. Arch Ophthalmol. 1997;115(3):335-344.

8. Honavar SG, Goyal M, Majji AB, Sen PK, Naduvilath T, Dandona L. Glaucoma after pars plana vitrectomy and silicone oil injection for complicated retinal detachments. Ophthalmology. 1999;106(1):169-176; discussion 177.

9. Errera MH, Liyanage SE, Elgohary M, et al. Using spectral-domain optical coherence tomography imaging to identify the presence of retinal silicone oil emulsification after silicone oil tamponade. Retina. 2013;33(8):1567-1573.

10. Heidenkummer HP, Messmer EM, Kampik A. Recurrent vitreoretinal membranes in intravitreal silicon oil tamponade. Morphologic and immunohistochemical studies. Ophthalmologe. 1996;93(2):121-125.

11. Wickham LJ, Asaria RH, Alexander R, Luthert P, Charteris DG. Immunopathology of intraocular silicone oil: retina and epiretinal membranes. Br J Ophthalmol. 2007;91(2):258-262.

12. Chung J, Spaide R. Intraretinal silicone oil vacuoles after macular hole surgery with internal limiting membrane peeling. Am JOphthalmol. 2003; 136(4):766-767.

13. Smiddy WE, Maguire AM, Green WR, et al. Idiopathic epiretinal membranes. Ultrastructural characteristics and clinicopathologic correlation. Ophthalmology. 1989;96(6):811-820; discussion 821.

14. Okada M, Ogino N, Matsumura M, Honda Y, Nagai Y. Histological and immunohistochemical study of idiopathic epiretinal membrane. Ophthalmic Res. 1995;27(2):118-128.

15. Michels RG. A clinical and histopathologic study of epiretinal membranes affecting the macula and removed by vitreous surgery. Trans Am Ophthalmol Soc. 1982;80:580-656.
Clinical Ophthalmology

\section{Publish your work in this journal}

Clinical Ophthalmology is an international, peer-reviewed journal covering all subspecialties within ophthalmology. Key topics include: Optometry; Visual science; Pharmacology and drug therapy in eye diseases; Basic Sciences; Primary and Secondary eye care; Patient Safety and Quality of Care Improvements. This journal is indexed on

Submit your manuscript here: http://www.dovepress.com/clinical-ophthalmology-journal

\section{Dovepress}

PubMed Central and CAS, and is the official journal of The Society of Clinical Ophthalmology (SCO). The manuscript management system is completely online and includes a very quick and fair peer-review system, which is all easy to use. Visit http://www.dovepress.com/ testimonials.php to read real quotes from published authors. 